\title{
TET1 Modulates H4K16 Acetylation by Interacting with hMOF to Regulate Expression of DNA Repair Genes and Oncogenic \\ Transformation
}

Jianing Zhong ${ }^{1}$, Xianfeng $\mathrm{Li}^{2,3}$, Wanshi $\mathrm{Cai}^{2,3}$, Yan Wang ${ }^{2,3}$, Shanshan Dong ${ }^{1}$, Jian'an Zhang $^{1}$, Jie Yang ${ }^{1}$, Kangli Wang ${ }^{1}$, Fengbiao $\mathrm{Mao}^{2,3}$, Cheng Zeng ${ }^{2,3}$, Yuanyuan $\mathrm{Li}^{2,3}$, Jinyu $\mathrm{Wu}^{2,3}$, Huanming Yang ${ }^{1}$, Xingzhi $\mathrm{Xu}^{4}$ and Zhong Sheng Sun ${ }^{1,2,3, *}$

${ }^{1}$ Institute of Genomic Medicine, Wenzhou Medical University, Wenzhou 325035, China

${ }^{2}$ Beijing Institutes of Life Science, Chinese Academy of Sciences, Beijing 100101, China

${ }^{3}$ University of Chinese Academy of Sciences, Beijing 100049, China

${ }^{4}$ Beijing Key Laboratory of DNA Damage Response and College of Life Sciences, Capital Normal University, Beijing 100049, China

* Correspondence: Zhong Sheng Sun: sunzs@mail.biols.ac.cn 


\section{ABSTRACT}

The Ten Eleven Translocation 1 (TET1) protein is a DNA demethylase that regulates gene expression through alteration of DNA methylation. Recent studies have demonstrated that TET1 could modulate transcriptional expression independent of its DNA demethylation activity; however, the detailed mechanisms underlying TET1's role in such transcriptional regulation remain not well understood. Here, we uncovered that Tet 1 formed a chromatin complex with histone acetyltransferase Mof and scaffold protein Sin3a in mouse embryonic stem cells by integrative genomic analysis using publicly available ChIP-seq data sets. Specifically, the TET1/SIN3A/hMOF complex mediates acetylation of histone $\mathrm{H} 4$ at lysine 16 , via facilitating the binding of hMOF on chromatin, to regulate expression of important DNA repair genes in DNA double strand breaks, including TP53BP1, RAD50, RAD51, and $B R C A 1$, for homologous recombination and non-homologous end joining repairs. Under hydrogen peroxide-induced DNA damage, dissociation of TET1 and hMOF from chromatin, concurrent with increased binding of SIRT1 on chromatin, led to hypo-acetylation of $\mathrm{H} 4 \mathrm{~K} 16$, reduced expression of these DNA repair genes, and DNA repair defects in a DNA methylation independent manner. A similar epigenetic dynamic alteration was also observed in $\mathrm{H}-\mathrm{RAS}^{\mathrm{V} 12}$ oncogenic-transformed cells, supporting the notion that suppression of TET1 downregulates DNA repair genes through modifying $\mathrm{H} 4 \mathrm{~K} 16 \mathrm{ac}$, instead of its demethylation function, and therefore contribute to tumorigenesis. Taken together, our results suggested a mechanistic link between a novel TET1 complex and H4K16ac, DNA repair genes expression, and genomic instability.

Keywords: TET1; H4K16 acetylation; dynamic regulation; DNA repair; oncogenic transformation 


\section{INTRODUCTION}

The Ten Eleven Translocation 1 (TET1) protein, a member of TET family, is a key player in DNA demethylation(Veron and Peters 2011). However, a recent study revealed that Tet1, in addition to its transcriptional regulatory function through its catalytic activity in DNA demethylation, possesses both activation and repressor functions in the regulation of a certain subset of genes in mouse embryonic stem cells (mESCs)(Williams et al. 2011). This observation was further supported by a study in which changes of transcriptional expression induced by overexpression of TET1 were highly similar to those induced by its demethylation-enzymatically-dead mutant in differentiated cell lines, suggesting that TET1 mainly regulates gene expression through a DNA methylation independent manner(Jin et al. 2014). The repressive role of TET1 in transcriptional regulation has been proposed to derive from its interaction with polycomb repressive complex 2 (PRC2) to form a histone modifying complex, thereby modifying chromatin repressive mark (H3K27me3) in mESCs(Wu et al. 2011). However, the interaction between TET1 and PRC2 complex is, so far, exclusively presented in embryonic stem cells (ESCs), but not in differentiated cells such as fibroblasts and HEK293T cells(Neri et al. 2013), indicating that TET1/PRC2 complex may act to repress gene expression in an ESCs-specific manner. On the other hand, SIN3A (homolog of $\operatorname{Sin} 3$ in yeast), a key component in multiple regulatory complexes, is involved in both transcriptional repression and activation through recruitment of diverse transcriptional factors or chromatin remodeling machinery at target promoters(Kadamb et al. 2013; Solaimani et al. 2014). A recent study has shown that TET1 interacts with SIN3A in both mESCs and HEK293T cells and presents highly overlapping binding profile on genome-wide(Williams et al. 2011), implying TET1 may associate with SIN3A to regulate gene expression in both ESCs and differentiated cells. However, the exact mechanisms underlying the functional nature of TET1 and its associated protein complexes in regulating its target gene expression remain to be unveiled.

Recently, it was demonstrated that there are dysfunctional DNA repair mechanisms and increased mutation frequencies in TET1-deficient non-Hodgkin B 
cell lymphoma (B-NHL), indicating that TET1 function as a tumor suppressor(Cimmino et al. 2015). This observation, in line with a previous study in which there were decreased foci of MLH1 and delayed removal of RAD51 in mouse Tet1-knockout primordial germ cells(Yamaguchi et al. 2012), indicates that TET1 plays an important role in DNA repair in mammalian cells. However, the underlying mechanisms of TET1 functions in DNA repair in response to DSBs are unclear.

Homologous recombination repair (HRR) and non-homologous end joining (NHEJ) are two categories of DNA repair pathway in response to DNA double strand breaks (DSBs). Some DNA repair genes, such as RAD50, BRCA1, RAD51, and TP53BP1, act as tumor suppressors and are frequently mutated or aberrantly downregulated in human cancers, resulting in impairments of DNA repair in response to DSBs, which is recognized as one of the hallmarks of tumorigenesis (Hanahan and Weinberg. 2011 ; Negrini et al. 2010). Whole Genome Bisulfate Sequencing (WGBS) data analysis in the Tet1-deficient primordial germ cells showed that the methylation levels of most DNA repair genes had no obvious alteration (Yamaguchi et al. 2012), indicating that Tet1 possibly affects expression of DNA repair genes through a mechanism independent of its DNA demethylation function.

$\mathrm{H} 4 \mathrm{~K} 16 \mathrm{ac}$ is a well-known targeted epigenetic site for post-translational modification in transcriptional activation(Taylor et al. 2013). Human MOF (hMOF, also known as KAT8), a member of the MYST (Moz-Ybf2/Sas3-Sas2-Tip60) family of HATs, specifically modifies $\mathrm{H} 4 \mathrm{~K} 16 \mathrm{ac}$ and is frequently downregulated in various types of cancers, including medullo-blastomas, breast carcinomas, colorectal carcinoma, gastric cancer, and renal cell carcinoma(Cao et al. 2014; Pfister et al. 2008). Studies have shown that depletion of hMOF renders both a global reduction of $\mathrm{H} 4 \mathrm{~K} 16 \mathrm{ac}$ and DNA repair defects in budding yeast and mammal cells (Li et al. ; Sharma et al. 2010). In addition, overexpression of hMOF reverses silencing of certain tumor suppressor genes induced by H4K16 deacetylation (Kapoor-Vazirani et al. 2008). Conversely, SIRT1 has the ability to deacetylate H4K16ac (Vaquero et al. 2007; Mishra et al. 2014), and is required for DNA repair and genomic stability in both yeast and mammals(Uhl et al. 2010; Boulton and Jackson 1998). Noteworthy, an elevated 
SIRT1 expression has been observed in a variety of human cancers relative to their non-transformed counterparts, including leukemia, glioblastoma, prostate, colorectal, and skin cancers(Chen et al. 2005; Huffman et al. 2007; Liu et al. 2009). Importantly, exogenous expression of SIRT1 reverses the effects of hMOF on H4K16ac and sensitization to the topoisomerase II inhibitor of cancer cells(Hajji et al. 2010), implying H4K16ac is dynamically modulated by both hMOF and SIRT1.

In this study, we first revealed, through integrative genomic analysis using publicly available ChIP-seq data sets, that significantly overlapped distribution of TET1, Sin3a, Mof, and H4K16ac were observed in mESCs. We further demonstrated that TET1, hMOF, and SIN3A interact with each other by in vitro biochemical studies in human cell lines. We next showed that the identified TET1 chromatin complex specifically modulates H4K16ac. Finally, we uncovered, under DNA damage and oncogenic-induced transformation, that dynamic recomposition of these TET1/SIN3A/hMOF chromatin complex components could cause hypo-acetylation of H4K16, thereby impairing DNA repair and ultimately involving in tumorigenesis in a DNA methylation independent manner. 


\section{RESULTS}

Integrative genomic analysis reveals similar binding patterns between Tet1, Mof, and H4K16ac in mESCs

Previous studies have generated a considerable number of ChIP-seq data sets of DNA binding proteins (DBPs) from mESCs, which is available in GEO and ENCODE databases. We retrieved all 219 available ChIP-seq data sets corresponding to 103 different DBPs in mESCs to investigate the co-occupancy between Tet1 and the rest of the DBPs (Supplementary Table. 1). In hierarchical clustering, pair-wise correlation analysis between Tet1 and other DBPs demonstrated that Tet1 could be grouped into one sub-cluster with 13 DBPs in 2000bp transcriptional start site (TSS) flanking region (defined as promoter) (Fig. 1a). Next, the same correlation analysis of each component in this sub-cluster and six available histone modifications in mESCs showed that Tet1, with other five DBPs (Kdm2a, Mof, Dpy30, Sin3a, and Lsd1), were closely related to H4K16ac, H3K4me3, H3K9ac, and H3K27ac (Fig. 1b). Given that Mof is the only histone acetyltransferase in the sub-cluster, whereas Kdm2a, Lsd1, and Dpy30 are either histone demethylases or histone methyltransferase complex component, we decided to focus on the investigation of the co-efficiency among the three histone acetylation marks and Tet1, Mof, and Sin3a by correlation analysis. Our results revealed that Tet1, Sin3a, Mof, and H4K16ac were clustered together with the highest correlation coefficients (Fig. 1c). Furthermore, ChIP-seq signals enrichment analysis revealed that Tet1, Sin3a, Mof, and H4K16ac had highly overlapping distribution patterns around promoter regions (Fig. 1d and Supplementary Fig. 1). Taken together, these observations imply that TET1, SIN3A, hMOF have highly similar binding patterns with $\mathrm{H} 4 \mathrm{~K} 16 \mathrm{ac}$ at the genomic level.

In order to determine whether TET1/SIN3A/hMOF was a complex distinguished from either PRC2 complex or SIN3A/HDAC complex and targeted different chromatin marks, we investigated the binding profiles of the major components of these three complexes, including Suz12, Ezh2, Sin3a, Tet1, Mof, Hdac1, and Hdac2, as well as their associated histone marks H3K27me3 and H4K16ac. Initially, we determined binding states by dividing promoter regions from ChIP-seq data set of each of above 
proteins and histone marks into clusters M1-M7 using ChromHMM (Supplementary Fig. 2a, 2b). Our results showed that Tet1/Sin3a/Mof complex, PRC2 complex, and Sin3a/Hdac1/Hdac2 complex were enriched in cluster M6, M7, and M3, respectively (Fig. 1e). Remarkably, Tet1, Mof, and Sin3a combined with H4K16ac, commonly enriched in cluster M6, was primarily related to DNA damage and repair associated pathways by KEGG pathway analysis (Fig. 1f). In addition, H4K16ac was enriched in M5, which was significantly associated with DNA repair related biological processes similar to those in M6 (Supplementary Fig. 2c). Intriguingly, we observed most of promoter regions in 177 DNA repair genes, including Brca1, Brca2, Rad51,Trp53, and Mlh1, as shown in Supplementary Fig. 3, were co-occupied with binding of Tet1, Sin3a, Mof, and H4K16ac. In summary, our integrated genomic analysis indicates that Tet1 may form a complex with Mof and Sin3a targeting H4K16ac in mESCs.

\section{TET1 forms a chromatin complex with SIN3A and hMOF to target H4K16ac}

To confirm that TET1 formed a chromatin complex with hMOF and SIN3A, we first obtained different fractions of nuclear protein extracts in HEK293T cells separated by size fractionation using sucrose gradient centrifugation. As shown in our data, TET1, hMOF, and SIN3A were simultaneously enriched in pool 3, 4, and 5, suggesting they may be complexed with each other (Fig. 2a). Next, we performed immunoprecipitations (IPs) analysis of chromatin-bound protein after overexpression of Flag-TET1 or HA-SIN3A in HEK293T cells. Our data showed that both Flag-TET1 and HA-SIN3A interacted with hMOF (Fig. 2b). In order to identify which region of TET1 interacted with hMOF and SIN3A, we constructed three fragment plasmids of TET1, described as Flag-FL1, Flag-FL2, and Flag-FL3, which respectively contained CXXC domain, Cysteine-rich domain and DSBH (double stranded $\beta$-helix) conserved domain (Fig. 2c). Our Co-IP data showed that hMOF and SIN3A only interacted with Flag-FL3 (Fig. 2d). Consistently, the interactions were confirmed by His-pulldown assays using proteins overexpressed in, and purified from E. coli cells (Fig. 2e). Taken together, our results suggest that TET1 forms a chromatin complex with hMOF and SIN3A via its C-terminal region. 
To determine whether TET1 targeted H4K16ac, we performed IP assays in HEK293T cells co-transfected with Flag-FL and HA-H4K16WT, HA-H4K16Q (a mimic acetylated mutant), or HA-H4K16R (an unacetylated mutant), respectively. Compared with interaction with HA-H4K16WT, Flag-FL had increased interaction with HA-H4K16Q, but decreased association with HA-H4K16R (Fig. 2f). Next, we performed chromatin fractionation and Western blot analysis on chromatin extracts from HEK293T cells with overexpressed HA-H4K16R and HA-H4K16WT, respectively. TET1 binding was significantly decreased in cells overexpressing HA-H4K16R compared with that in cells overexpressing HA-H4K16WT (Fig. 2g). In addition, when HEK293T cells were treated with the HDAC class I and class II inhibitor trichostatin A (TSA), increased TET1 binding was observed (Fig. 2h). Taken together, our data indicates that TET1 preferentially associates with histone $\mathrm{H} 4$ bearing $\mathrm{K} 16$ acetylation mark.

\section{TET1 depletion causes a significant reduction in H4K16ac levels}

Given that TET1 contains CXXC domain, which enable its direct DNA binding, we reason that TET1 may recruit hMOF to genomic loci for regulation of H4K16ac mark. To test this hypothesis, we analyzed alterations of several histone modifications in TET1-depleted cells. Western blot analysis showed that H4K16 was hypo-acetylated in Tet1-knockout mice embryonic fibroblast (Tet1 ${ }^{-/}$MEF) cells compared to in wild type Tet $1^{+/+}$MEFs. We observed a similar hypo-acetylation status in TET1-knockdown HCT116 and HeLa cells, respectively. However, the other histone markers, such as H3K4me2, H3K4me3, and H3K27me3, demonstrated insignificant alterations (Fig. 3a). Immunofluorescence staining further confirmed a significant reduction of H4K16ac in Tet1 ${ }^{-/-}$MEF cells, TET1-knockdown HCT116, and TET1-knockdown HeLa cells compared to their respective controls. Our results indicated that depletion of TET1 resulted in a significant reduction of H4K16ac level (Fig. 3b). Agree with this observation, the level of chromatin bound hMOF was significantly decreased in TET1-knockdown HEK293T cells (Fig. 4a). 
As hypo-acetylation of $\mathrm{H} 4 \mathrm{~K} 16$ was reported to facilitate 53BP1 recruitment(Hsiao and Mizzen), we also determined the number of 53BP1 foci and found a two-fold increase in the number of 53BP1 foci in Tet $1^{-1-}$ MEF cells (Fig. $\mathbf{3 c}$ and $\mathbf{3 d}$ ). Our results indicate that depletion of TET1 results in a significant reduction of H4K16ac levels and promotion the recruitment of 53BP1 binding to chromatin.

\section{TET1 switches binding of hMOF and/or SIRT1 on chromatin to regulate H4K16ac mark on the promoters of the DNA repair genes}

Interestingly, using chromatin fractionation we found SIRT1 binding increased in TET1-knockdown HEK293T cells, whereas hMOF binding was decreased (Fig. 4a), indicating that TET1, as a switch, controls bindings of hMOF and SIRT1 to chromatin. To determine whether TET1 was required for regulation of DNA repair genes through modifying H4K16ac, we measured the mRNA levels of RAD50, BRCA1, RAD51, and TP53BP1 in HA-hMOF overexpression or SIRT1-knockdown HEK393T cells, with or without TET1 knockdown, via RT-qPCR. We found that depletion of TET1 blocked the increased expression of RAD50, BRCA1, RAD51, and TP53BP1 both in hMOF-overexpressing cells (Fig. 4b) and in SIRT1-knockdown cells (Fig. 4d). Next, we further determined the effect of $\mathrm{H} 4 \mathrm{~K} 16 \mathrm{ac}$ enrichment at the promoter of RAD50, BRCA1, RAD51, and TP53BP1 in HA-hMOF-overexpressing or SIRT1-knockdown HEK293T cells, with or without TET1 knockdown, via ChIP-qPCR. The results showed that depletion of TET1 blocked the increased H4K16ac enriched at the promoter of RAD50, BRCA1, RAD51, and TP53BP1 in both hMOF-overexpressing (Fig. 4c) and SIRT1-knockdown HEK293T cells (Fig. 4e). These results suggest that TET1, as a switch, inversely modulates the bindings between hMOF and SIRT1 on chromatin, which in turn dynamically controls H4K16ac levels, and ultimately contributes to the expression of these important DNA repair genes.

Impairment of homologous recombination repair and non-homologous end joining in TET1-, but not TET2- or TET3-knockdown cells 
To determine the role of TET1 in DNA repair, C57 wild type mice (WT) and Tet1 heterozygous mutated mice $\left(T e t 1^{+/-}\right.$mice) were subjected to $\mathrm{x}$-ray radiation. The coat-state rating scale results showed that there was a severe deterioration of the coat in Tet $1^{+-}$mice compared with WT mice after four months of $\mathrm{x}$-ray radiation (Fig. $5 \mathbf{a}, \mathbf{5 b})$, suggesting that $T e 1^{+/-}$mice had defects in DNA repair mechanisms in response to DSBs. Next, we measured the percentage of DNA present in comet tail and the tail moment in comet assay to determine the extent of DNA damage in Tet $1^{-1-}$ MEF cells, respectively. Our results showed both of these parameters were increased approximately by two-folds in the Tet ${ }^{-1-}$ MEF cells than those in WT cells (Fig. $\mathbf{5 c}, \mathbf{5 d}$, and $\mathbf{5 e}$ ), indicating that Tet $1^{--}$MEF cells had a higher level of DSBs. We also measured foci formation of DSBs maker $\gamma \mathrm{H} 2 \mathrm{AX}$ by immunofluorescence staining. Consistent with our comet assay, we found that the number of $\gamma \mathrm{H} 2 \mathrm{AX}$ foci increased two-fold in Tet ${ }^{-1-}$ MEF cells compared to WT cells (Supplementary Fig4a, 4b). In addition, Western blot analysis indicated that the level of $\gamma \mathrm{H} 2 \mathrm{AX}$ increased in TET1-knockdown cells, but not in TET2- or TET3-knockdown HEK293T cells (Supplementary Fig.4c). DAPI staining and statistical analysis further showed that the percentage of micronuclei in Tet $1^{-/}$MEF cells were two-folds higher than that in WT cells (Fig. $\mathbf{5 f}, \mathbf{5 g}$ ). These results indicate that loss of TET1 leads to severe DNA damage and the defects in DNA repair and genomic instability.

Homologous recombination repair and non-homologous end joining are two types of DNA repair mechanism in response to DSBs. To determine the extent of HRR and NHEJ repair frequencies in TET1-depleted cells, we set out to employ two types of GFP reporter systems in HEK293T cells, in which a defective GFP gene is functionally restored to WT cells upon I-Scel transfection(Mao et al. 2008). We found TET1-depletion resulted in $25 \%$ decrease of GFP positive cell numbers in HRR reporter assay (Fig. $\mathbf{5 h}$ ), and $50 \%$ reduction in NHEJ reporter assay, and neither of these outcomes was observed in TET2- and TET3-knockdown cells (Fig. 5i). More importantly, we demonstrated that TET1-depletion induced transcriptional repression of the important genes in DNA repair, including $R A D 50, B R C A 1, R A D 51$, and TP53BP1, via RT-PCR and Western blot analysis (Fig. 5j, 5k). Collectively, our results 
indicate that depletion of TET1, but not TET2 or TET3, results in the defects of HRR and NHEJ in response to DSBs and genomic instability through downregulation of the DNA repair genes.

\section{Dynamic regulation of TET1, SIN3A, hMOF, and SIRT1 binding on chromatin in response to hydrogen peroxide-induced oxidative damage}

Next, we investigated alterations in different histone modifications by treating HEK293T cells with DNA damaging reagents including adriamycin (ADR), bleomycin (Bleo), camptothecin (CPT), and hydrogen peroxide $\left(\mathrm{H}_{2} \mathrm{O}_{2}\right)$. Our data showed that $\mathrm{H}_{2} \mathrm{O}_{2}$ treatment specifically resulted in a significant decrease of $\mathrm{H} 4 \mathrm{~K} 16$ ac levels, whereas $\mathrm{H} 3 \mathrm{~K} 27 \mathrm{me} 3, \mathrm{H} 3 \mathrm{~K} 4 \mathrm{me} 2$, and $\mathrm{H} 3 \mathrm{~K} 4 \mathrm{me} 3$ had no obvious changes in response to DNA damage reagents (Fig. 6a). In addition, we measured the bindings of TET1, hMOF1, SIRT1, and SIN3A on chromatin after DNA damage reagent treatment as described above. We found that $\mathrm{H}_{2} \mathrm{O}_{2}$ treatment caused a significant decrease of both TET1 and hMOF binding, and an increase in SIRT1 binding, but no obvious alteration of SIN3A binding (Fig. 6b). To support this notion, $\mathrm{H}_{2} \mathrm{O}_{2}$ treatment caused significantly decreased interactions between Flag-FL3 with hMOF, but not with SIN3A (Fig. 6c). As shown in Fig. $6 \mathbf{d}, \mathrm{H}_{2} \mathrm{O}_{2}$ treatment also led to transcriptional repression of the important genes in DNA repair including RAD50, BRCA1, RAD51, and TP53BP1, while the DNA methylation level, as assessed by profiling using reduce representation bisulfite sequencing (RRBS), did not present alteration in their corresponding promoters (Fig. 6e, 6f). ChIP-qPCR assay further revealed that TET1 and H4K16ac enriched together at promoter regions of these DNA repair genes and displayed decreased enrichment after $\mathrm{H}_{2} \mathrm{O}_{2}$ treatment (Fig. 6g). Taken together, our results suggest that oxidative damage induces dynamic recomposition of TET1, hMOF, and SIRT1 binding on chromatin, which is concurrent with the deacetylation of H4K16 and reduced expression of the important DNA repair genes.

TET1 binding on chromatin is dependent on ataxia telangiectasia mutated (ATM) protein in HEK293T cells 
Our data also showed an increased TET1 binding on chromatin after wortmannin treatment, which is an inhibitor of early DNA damage response proteins PIKK family (phosphatidylinositol 3-kinase-related protein kinase) (Supplementary Fig. 5a), indicating that TET1 binding was dependent on PIKK family kinases. Further studies showed that there was increased binding of TET1 after ATM inhibition using either its inhibitor CGK733 or ATM-siRNA (Supplementary Fig. 5b, 5c), suggesting that binding of TET1 on chromatin is dependent on ATM, but not DNA-PKcs (Supplementary Fig. 5d).

\section{A similar epigenetic TET1/SIN3A/hMOF recomposition mechanism is verified in an H-RAS ${ }^{\mathrm{V} 12}$ transformed cell line}

Previous studies have reported that oxidative damage leads to a higher risk of cancers in humans by diminishing histone acetylation, which predominantly occur at H4K16ac(Leufkens et al. ; Fraga et al. 2005; Sosa et al. 2013). Therefore, we hypothesized that dynamic recomposition of this TET1/SIN3A/hMOF/SIRT1 chromatin components might contribute to tumorigenesis. By employing an epithelial ovarian cell line T29 and its oncogenic counterpart T29H cell line which is transformed with H-RAS ${ }^{\mathrm{V} 12}$, we simultaneously analyzed the levels of oxidation and H4K16ac, expression of DNA repair genes, and the binding of TET1, SIN3A, hMOF1, and SIRT1, as compared with those in WT cells. Consistently, transcriptional expression of RAD50, BRCA1, RAD51, and TP53BP1 was decreased in T29H cells (Fig. 7a), but the methylation levels on their promoters was not significantly altered according to our RRBS data (Supplementary Fig. 6a). As expected, compared to T29 cells, there were decreased bindings of TET1 and hMOF, and increased SIRT1 binding on chromatin in $\mathrm{T} 29 \mathrm{H}$ cells (Fig. 7b), concurrent with the reduction of $\mathrm{H} 4 \mathrm{~K} 16 \mathrm{ac}$ levels (Fig. 7c). The bindings of both TET1 and H4K16ac on the promoter of these DNA repair genes were decreased as well (Fig. 7d, 7e). Meanwhile, we also found elevated 8-OH-dG level using a dot blot assay in T29H cell (Supplementary Fig. 6b). These results suggested that oncogenic transformation causes hypoacetylation of H4K16 via decreased chromatin binding of TET1 and hMOF1, and increased binding 
bioRxiv preprint doi: https://doi.org/10.1101/024844; this version posted August 17, 2015. The copyright holder for this preprint (which was not certified by peer review) is the author/funder, who has granted bioRxiv a license to display the preprint in perpetuity. It is made available under aCC-BY 4.0 International license.

of SIRT1, which lead to downregulation of the DNA repair genes, ultimately impairing DNA repair and involving tumorigenesis. 


\section{DISCUSSION}

In this study, we revealed that TET1 could function as a core component of TET1/SIN3A/hMOF chromatin complex, supported by their co-occupancy of common targets across the genome, association with each other by co-IP, and co-migration in size fractionation assays (Fig. 1, 2a-e). However, we still cannot rule out the possibility that TET1, hMOF, and SIN3A form sub-complexes to co-occupy the same genomic regions. We demonstrated that TET1 controlled the bindings of hMOF and SIRT1 on chromatin to specifically modulate alteration of $\mathrm{H} 4 \mathrm{~K} 16 \mathrm{ac} . \mathrm{H}_{2} \mathrm{O}_{2}$-induced oxidative damage could induce recomposition of this chromatin complex to provoke hypoacetylation of $\mathrm{H} 4 \mathrm{~K} 16$, which suppressed the expression of the important DNA repair genes and ultimately impaired HRR and NHEJ. Consistently, by employing H-RAS ${ }^{V 12}$ oncogenic transformed cell line, we revealed a similar epigenetic recomposition mechanism for understanding the role of TET1 in tumorigenesis. With this work, a novel role of TET1 is proposed as shown in Fig. 7f.

Previous reports showed TET1 and modified 5-hmC correlates with "bivalent" chromatin markers with both repressing (H3K27me3) and activating (H3K4me3) chromatin markers in mESCs(Pastor et al. 2011 ; Wu et al. 2011). The fact that TET1 contributes to silencing some genes by facilitating recruitment of PRC2 complex( $\mathrm{Wu}$ et al. 2011), supports the hypothesis the TET1 represses gene expression by forming a TET1/PRC2 repressor complex that targets H3K27me3. However, the following two lines of evidence imply that involvement of TET1/PRC2 in transcriptional repression may be ESCs-specific: 1) correlation between $5 \mathrm{hmC}$ and $\mathrm{H} 3 \mathrm{~K} 27 \mathrm{me} 3$ is unique to ESCs, and is not present in differentiated fibroblasts or adult tissues; and 2) interaction between TET1 and the components of PRC2 complex were only observed in ESCs, but not in fibroblasts or HEK293T cells(Neri et al. 2013). Our observation, in which co-occupancy between Tet1 and Suz12/Ezh2/H3K27me3 present in cluster M7 (mainly involved in cell differentiation and cell fate functions) (Supplementary Fig. 2c), further supports the notion that Tet1/PRC2 complex may play an important role in cell differentiation and cell fate in mESCs. Additionally, we observed high co-occupancy of Tet1/Mof/Sin3a/H4K16ac at DNA repair genes in cluster M6, 
whereas binding of H3K27me3 was absent (Fig.1). Our results, combined with a previous report in which TET1 was shown to interact with SIN3A in HEK293 cells and their shared binding on TSS region are H3K27me3-binding negative(Neri et al. 2013), exclude the possibility that TET1 downregulates these DNA repair genes by TET1/PRC2 complex through activating the repressive histone mark H3K27me3 in differentiated cells.

Recent studies demonstrate that TET1 acts as a stable component of O-GlcNAc transferase (OGT) in ESCs, and promotes histone O-GlcNAcylation and H3K4me3 states for gene activation(Vella et al. 2013). However, several reports argue that TET2 and TET3, but not TET1, interact with OGT to activate gene expression in HEK293T cells(Chen et al. 2005; Deplus et al. 2013), raising the possibility that TET1/OGT complex might be involved in transcriptional activation exclusively in ESCs. Our results in several differentiated cells lines revealed that TET1 knockdown resulted in hypoacetylation of H4K16 only, but did not affect levels of H3K27me3, H3K4me2, and H3K4me3, suggesting that TET1 complex mainly modulate H4K16ac in these cells (Fig.3). Interestingly, the observations that inhibition of HDAC1/HDAC2 has no significant effect on some important genes in DNA repair, including RAD50 and BRCA1(Thurn et al. 2013), and HDAC1/HDAC2 do not have occupancy on the promoter of $R A D 50, B R C A 1, R A D 51$, and TP53BP1 in ChIP-seq data from mESCs (Supplementary Table 2), imply that TET1/SIN3A may not form a complex with HDAC1/HDAC2 to repress these DNA repair genes.

One of main etiological hypothesis linking genomic instability, mutagenesis and tumorigenesis is that deficient DNA repair mechanisms is derived from extensive oxidative DNA damage and cellular injury(Ziech et al. 2011; Gencer et al. 2012). The relationship between DNA damage and epigenetic gene silencing has been examined using an engineered cell model, in which an I-Scel restriction site was integrated into the $\mathrm{CpG}$ island of the E-cadherin promoter(O'Hagan et al. 2008). That study showed that $\mathrm{H}_{2} \mathrm{O}_{2}$-induced oxidative damage regulated epigenetic DNA methylation changes through redistribution of DNMT1, EZH2, and SIRT1 on chromatin from non-CpG rich regions to $\mathrm{CpG}$ islands $\left(\mathrm{O}^{\prime} \mathrm{Hagan}\right.$ et al. 2011). Here, we observed that $\mathrm{H}_{2} \mathrm{O}_{2}$-induced 
oxidative damage directly resulted in 165 differentially methylated regions (DMRs) in promoters (data not shown); however, none of these involved in genes in the DNA repair pathway (Fig. 6). These results further support that downregulation of expression of RAD50, BRCA1, RAD51, and TP53BP1 under $\mathrm{H}_{2} \mathrm{O}_{2}$-induced oxidative damage, is independent of promoter methylation changes on those genes. Interestingly, we show that SIRT1 binding is elevated under both $\mathrm{H}_{2} \mathrm{O}_{2}$-induced oxidative damage and oncogenic transformation, and SIRT1 forms a chromatin complex with PRC2 complex subunits in response to $\mathrm{H}_{2} \mathrm{O}_{2}$-induced oxidative damage, which is consistent with a previous report(O'Hagan et al. 2011) (Supplementary Fig. 7a). This supports the hypothesis that epigenetic silencing of important genes in DNA repair both in cancer cells and under oxidative damage, likely results from loss of TET1 which switches the bindings of hMOF and SIRT1 on chromatin, thus promoting aberrant hypoacetylation of $\mathrm{H} 4 \mathrm{~K} 16$, rather than the alteration of DNA methylation. This hypothesis is further supported by the observation that a similar epigenetic dynamic alteration occurred in $\mathrm{H}-\mathrm{RAS}^{\mathrm{V} 12}$ oncogenic transformed cells (Fig. 7). Stephen P. Jackson's group showed that H3K4me3, H3K18ac, H4K5ac, and $\mathrm{H} 4 \mathrm{~K} 12 \mathrm{ac}$ were unaffected in response to oxidative damage(Tjeertes et al. 2009). Our results also suggested that it's unlikely that hypoacetylation of $\mathrm{H} 3 \mathrm{~K} 9 \mathrm{ac}$ plays a repressive role in regulation of important genes in DNA repair in response to $\mathrm{H}_{2} \mathrm{O}_{2}$-induced oxidative damage (Supplementary Fig. 6b). These evidence suggest that hypoacetylation of $\mathrm{H} 4 \mathrm{~K} 16$ is likely a major mechanism to downregulate these DNA repair genes under oxidative damage. However, the repressive role of other histone marks and microRNAs cannot be ruled out and need to be further explored. 


\section{MATERIALS and METHODS}

\section{Mice}

Tet $1^{+/-}$mice are obtained from the Jackson Laboratory (Cat\# 017358). For genotyping of Tet $^{+/-}$mice, the forward primer AACTGATTCCCTTCGTGCAG, and the reverse primer TTAAAGCATGGGTGGGAGTC were used. The expected band size for homozygote mutant was $650 \mathrm{bp}$, $850 \mathrm{bp}$ for the wild type strain, and $650 \mathrm{bp}$ and $850 \mathrm{bp}$ double bands for the heterozygote strain.

\section{X-ray irradiation}

Irradiations were performed at the Chinese Academy of Sciences (Beijing)using an x-ray machine (RS-2000 PRO Biological system). WT mice and Tet1 $1^{+-}$mice were irradiated with a single whole-body dose of 3Gy x-ray at 60 days of age. The irradiation was operating at $160-\mathrm{kV}$ constant potential and $25 \mathrm{~mA}(0.3 \mathrm{~mm}$ Cu filter) at a dose rate equal to $1.136 \mathrm{~Gy} \min -1$ for a total of $2.64 \mathrm{~min}$. The cage was cleaned with $75 \%$ ethanol when each of irradiation was finished. Coat-state condition of WT mice and $T e t 1^{+/}$mice with irradiation or sham-irradiated were scored after four month of irradiation.

\section{Cell Culture, Plasmids, and siRNA Oligonucleotides}

Mouse Tet $1^{-/-}$MEF cells were a generous gift from Dr. Guoliang Xu, SIBS of Chinese Academy of Sciences, Shanghai. T29 and T29H cells were a generous gift from Dr. Jason Liu from MD Anderson Cancer Center of University of Texas, Houston. All the cells were cultured in DMEM media (Hyclone, USA), supplemented with $10 \%$ fetal bovine serum (FBS; Hyclone, USA) and penicillin-streptomycin (Invitrogen, USA). H4K16Q and H4K16R mutants were generated using a QuickChange Multi Site-Directed Mutagenesis Kit (Stratagene, USA). RNA interference (RNAi) experiments were performed using Dharmacon siGENOME SMARTpool siRNA duplexes (Thermo Fisher Scientific, USA) against TET1, TET2, TET3, HDAC1, HDAC2, ATM, and SIRT1 respectively. TET1 cDNA was purchased from Origene (RC218608), cDNA for hMOF, SIRT1, SIN3A, and Histone H4 were generated from 
cDNA library and subcloned into pcDNA3.0 vector, followed by sequencing validation. Antibodies used in this study were purchased from different commercial companies as detailed in Supplemental Methods.

siRNA Transfection, RNA Isolation, and qRT-PCR

See Supplemental Methods for detailed information.

\section{ChIP-seq Data Preparation}

For integrated genomic analysis, we collected 219 ChIP-seq data sets of 103 DNA binding proteins and 14 data sets of 8 histone modification markers in mESCs from GEO and ENCODE (See Supplementary Table 1). Further analysis details are provided in Supplemental Methods.

\section{RRBS Library Preparation, Sequencing and Analysis}

See Supplemental Methods for detailed information.

\section{ASSESSION}

\section{GSE66395}




\section{AUTHOR CONTRIBUTIONS}

J.N.Z. and Z.S.S. designed the experiments; S.S.D. and J.A.Z. performed immunofluorescence and microscopic analysis; K.L.W. helped W.S.C. with ChIP-Seq and RRBS; X.F.L. and F.B.M. performed ChIP-Seq and RRBS data analysis; S.S.D. and J.A.Z. helped for immunoprecipitation and cellular fractionation experiments. J.Y. helped for gene cloning. Z.C, Y.Y.L. and Y.W. helped for mice experiments. J.N.Z. and Z.S.S. wrote the manuscript; H.M.Y., X.Z.X. and J.Y.W. revised the manuscript.

\section{ACKNOWLEDGMENTS}

We are grateful to Dr. Guoliang Xu for a kind gift of TET1 knockout MEF cells, Dr. Jie Du for critically evaluating the manuscript, and Dr. Xingda Ju and the other members in our lab for help in experiments. This work is supported by National High Technology Research and Development Program of China (No. 2012AA02A202); Natural Science Foundation of China (NSFC)-Canadian Institutes of Health Research Collaborative Research Project (No. 81161120541); Nation Natural Science Foundation of China (NSFC)(No. 81301778); Zhejiang Provincial Natural Science Foundation (ZNSF) (No. LY13C060002); Zhejiang Provincial Department of Education Research Project (No. 84612051); Nation Natural Science Foundation of China (NSFC) (No. 31271266); Startup Fund for scholars of Wenzhou medical university (No. QTJ12001), and grant from Innovative Center of China, AstraZeneca.

\section{COMPETING FINANCIAL INTERESTS}

The authors declare no competing financial interests. 


\section{REFERENCES}

Boulton SJ, Jackson SP. 1998. Components of the Ku-dependent non-homologous end-joining pathway are involved in telomeric length maintenance and telomeric silencing. The EMBO journal 17(6): 1819-1828.

Cao L, Zhu L, Yang J, Su J, Ni J, Du Y, Liu D, Wang Y, Wang F, Jin J et al. Correlation of low expression of hMOF with clinicopathological features of colorectal carcinoma, gastric cancer and renal cell carcinoma. International journal of oncology 44(4): 1207-1214.

Chen Q, Chen Y, Bian C, Fujiki R, Yu X. TET2 promotes histone O-GlcNAcylation during gene transcription. Nature 493(7433): 561-564.

Chen WY, Wang DH, Yen RC, Luo J, Gu W, Baylin SB. 2005. Tumor suppressor HIC1 directly regulates SIRT1 to modulate p53-dependent DNA-damage responses. Cell 123(3): 437-448.

Cimmino L, Dawlaty MM, Ndiaye-Lobry D, Yap YS, Bakogianni S, Yu Y, Bhattacharyya S, Shaknovich R, Geng H, Lobry C et al. 2015. TET1 is a tumor suppressor of hematopoietic malignancy. Nature immunology.

Deplus R, Delatte B, Schwinn MK, Defrance M, Mendez J, Murphy N, Dawson MA, Volkmar M, Putmans $\mathrm{P}$, Calonne $\mathrm{E}$ et al. TET2 and TET3 regulate GlcNAcylation and H3K4 methylation through OGT and SET1/COMPASS. The EMBO journal 32(5): 645-655.

Fraga MF, Ballestar E, Villar-Garea A, Boix-Chornet M, Espada J, Schotta G, Bonaldi T, Haydon C, Ropero S, Petrie K et al. 2005. Loss of acetylation at Lys16 and trimethylation at Lys20 of histone $\mathrm{H} 4$ is a common hallmark of human cancer. Nature genetics 37(4): 391-400.

Gencer M, Dasdemir S, Cakmakoglu B, Cetinkaya Y, Varlibas F, Tireli H, Kucukali CI, Ozkok E, Aydin M. 2012. DNA repair genes in Parkinson's disease. Genetic testing and molecular biomarkers 16(6): 504-507.

Hajji N, Wallenborg K, Vlachos P, Fullgrabe J, Hermanson O, Joseph B. 2010. Opposing effects of hMOF and SIRT1 on H4K16 acetylation and the sensitivity to the topoisomerase II inhibitor etoposide. Oncogene 29(15): 2192-2204. 
Hanahan D, Weinberg RA. Hallmarks of cancer: the next generation. Cell 144(5): 646-674.

Hsiao KY, Mizzen CA. Histone H4 deacetylation facilitates 53BP1 DNA damage signaling and double-strand break repair. Journal of molecular cell biology $5(3)$ : 157-165.

Huffman DM, Grizzle WE, Bamman MM, Kim JS, Eltoum IA, Elgavish A, Nagy TR. 2007. SIRT1 is significantly elevated in mouse and human prostate cancer. Cancer research 67(14): 6612-6618.

Jin C, Lu Y, Jelinek J, Liang S, Estecio MR, Barton MC, Issa JP. TET1 is a maintenance DNA demethylase that prevents methylation spreading in differentiated cells. Nucleic acids research 42(11): 6956-6971.

Kadamb R, Mittal S, Bansal N, Batra H, Saluja D. 2013. Sin3: insight into its transcription regulatory functions. European journal of cell biology 92(8-9): 237-246.

Kapoor-Vazirani P, Kagey JD, Powell DR, Vertino PM. 2008. Role of hMOF-dependent histone $\mathrm{H} 4$ lysine 16 acetylation in the maintenance of TMS1/ASC gene activity. Cancer research 68(16): 6810-6821.

Leufkens AM, van Duijnhoven FJ, Woudt SH, Siersema PD, Jenab M, Jansen EH, Pischon T, Tjonneland A, Olsen A, Overvad $\mathrm{K}$ et al. Biomarkers of oxidative stress and risk of developing colorectal cancer: a cohort-nested case-control study in the European Prospective Investigation Into Cancer and Nutrition. American journal of epidemiology 175(7): 653-663.

Li X, Corsa CA, Pan PW, Wu L, Ferguson D, Yu X, Min J, Dou Y. MOF and H4 K16 acetylation play important roles in DNA damage repair by modulating recruitment of DNA damage repair protein Mdc1. Molecular and cellular biology 30(22): 5335-5347.

Liu T, Liu PY, Marshall GM. 2009. The critical role of the class III histone deacetylase SIRT1 in cancer. Cancer research 69(5): 1702-1705. 
Mao Z, Bozzella M, Seluanov A, Gorbunova V. 2008. DNA repair by nonhomologous end joining and homologous recombination during cell cycle in human cells. Cell cycle (Georgetown, Tex 7(18): 2902-2906.

Mishra BP, Zaffuto KM, Artinger EL, Org T, Mikkola HK, Cheng C, Djabali M, Ernst P. The Histone Methyltransferase Activity of MLL1 Is Dispensable for Hematopoiesis and Leukemogenesis. Cell reports 7(4): 1239-1247.

Negrini S, Gorgoulis VG, Halazonetis TD. Genomic instability--an evolving hallmark of cancer. Nature reviews 11(3): 220-228.

Neri F, Incarnato D, Krepelova A, Rapelli S, Pagnani A, Zecchina R, Parlato C, Oliviero S. 2013. Genome-wide analysis identifies a functional association of Tet1 and Polycomb repressive complex 2 in mouse embryonic stem cells. Genome biology 14(8): R91.

O'Hagan HM, Mohammad HP, Baylin SB. 2008. Double strand breaks can initiate gene silencing and SIRT1-dependent onset of DNA methylation in an exogenous promoter CpG island. PLoS genetics 4(8): e1000155.

O'Hagan HM, Wang W, Sen S, Destefano Shields C, Lee SS, Zhang YW, Clements EG, Cai Y, Van Neste L, Easwaran $\mathrm{H}$ et al. 2011. Oxidative damage targets complexes containing DNA methyltransferases, SIRT1, and polycomb members to promoter CpG Islands. Cancer cell 20(5): 606-619.

Pastor WA, Pape UJ, Huang Y, Henderson HR, Lister R, Ko M, McLoughlin EM, Brudno Y, Mahapatra S, Kapranov P et al. Genome-wide mapping of 5-hydroxymethylcytosine in embryonic stem cells. Nature 473(7347): 394-397.

Pfister S, Rea S, Taipale M, Mendrzyk F, Straub B, Ittrich C, Thuerigen O, Sinn HP, Akhtar A, Lichter P. 2008. The histone acetyltransferase hMOF is frequently downregulated in primary breast carcinoma and medulloblastoma and constitutes a biomarker for clinical outcome in medulloblastoma. International journal of cancer 122(6): 1207-1213.

Sharma GG, So S, Gupta A, Kumar R, Cayrou C, Avvakumov N, Bhadra U, Pandita RK, Porteus MH, Chen DJ et al. 2010. MOF and histone H4 acetylation at 
lysine 16 are critical for DNA damage response and double-strand break repair. Molecular and cellular biology 30(14): 3582-3595.

Solaimani P, Wang F, Hankinson O. 2014. SIN3A, generally regarded as a transcriptional repressor, is required for induction of gene transcription by the aryl hydrocarbon receptor. The Journal of biological chemistry 289(48): 33655-33662.

Sosa V, Moline T, Somoza R, Paciucci R, Kondoh H, ME LL. 2013. Oxidative stress and cancer: an overview. Ageing research reviews 12(1): 376-390.

Taylor GC, Eskeland R, Hekimoglu-Balkan B, Pradeepa MM, Bickmore WA. 2013. H4K16 acetylation marks active genes and enhancers of embryonic stem cells, but does not alter chromatin compaction. Genome research 23(12): 2053-2065.

Thurn KT, Thomas S, Raha P, Qureshi I, Munster PN. 2013. Histone deacetylase regulation of ATM-mediated DNA damage signaling. Molecular cancer therapeutics 12(10): 2078-2087.

Tjeertes JV, Miller KM, Jackson SP. 2009. Screen for DNA-damage-responsive histone modifications identifies H3K9Ac and H3K56Ac in human cells. The EMBO journal 28(13): 1878-1889.

Uhl M, Csernok A, Aydin S, Kreienberg R, Wiesmuller L, Gatz SA. Role of SIRT1 in homologous recombination. DNA repair 9(4): 383-393.

Vaquero A, Sternglanz R, Reinberg D. 2007. NAD+-dependent deacetylation of H4 lysine 16 by class III HDACs. Oncogene 26(37): 5505-5520.

Vella P, Scelfo A, Jammula S, Chiacchiera F, Williams K, Cuomo A, Roberto A, Christensen J, Bonaldi T, Helin $\mathrm{K}$ et al. Tet proteins connect the O-linked $\mathrm{N}$-acetylglucosamine transferase Ogt to chromatin in embryonic stem cells. Molecular cell 49(4): 645-656.

Veron N, Peters AH. 2011. Epigenetics: Tet proteins in the limelight. Nature 473(7347): 293-294. 
Williams K, Christensen J, Pedersen MT, Johansen JV, Cloos PA, Rappsilber J, Helin K. 2011. TET1 and hydroxymethylcytosine in transcription and DNA methylation fidelity. Nature 473(7347): 343-348.

Wu H, D'Alessio AC, Ito S, Xia K, Wang Z, Cui K, Zhao K, Sun YE, Zhang Y. 2011. Dual functions of Tet1 in transcriptional regulation in mouse embryonic stem cells. Nature 473(7347): 389-393.

Yamaguchi S, Hong K, Liu R, Shen L, Inoue A, Diep D, Zhang K, Zhang Y. 2012. Tet1 controls meiosis by regulating meiotic gene expression. Nature 492(7429): 443-447.

Ziech D, Franco R, Pappa A, Panayiotidis MI. 2011. Reactive oxygen species (ROS)--induced genetic and epigenetic alterations in human carcinogenesis. Mutation research 711(1-2): 167-173.

\section{FIGURE LEGENDS}

Figure 1. Integrative genomic analysis of published ChIP-seq data sets in mESCs show highly similar binding patterns between Tet1 and H4K16ac

(a) Pearson correlation between Tet1 and other 102 DBPs (Supplementary Table 1) in 2000bp TSS flanking regions. deepTools was employed to calculate the correlation of ChIP-seq data. Color bar represented correlation coefficient. (b) Further correlation between Tet1, other 13 DBPs (Kdm2a, Dpy30, Mof, Sin3a, Lsd1, Oct4, p300, Dax1, Dmap1, Max, Nanog, Myc, and Tip60) and 6 histone modifications (H3K4me3, H3K9me3, H3K9ac, H3K27ac, H3K36me3, and H4K16ac) in 2000bp TSS flanking regions. (c) Correlation of Tet1, Sin3a, Mof, H4K16ac, H3K27ac and H3K9ac. (d) The bindings of Tet1, Sin3a, Mof, and H4K16ac are commonly enriched in the TSS regions. (e) Seven distinct distribution among Tet1/Sin3a/Mof complex, PRC2 complex, and Sin3a/Hdac1/Hdac2 complex were generated by ChromHMM. (f) The enriched pathways in cluster M6, including DNA repair pathways marked as red color.

Figure 2. TET1 forms a chromatin complex with hMOF, and SIN3A targeting H4K16ac 
(a) Nuclear extracts from HEK293T cells were added to a $12 \%-30 \%$ sucrose gradient, and fractions were assayed by immunoblotting. The fraction numbers and $660 \mathrm{kDa}$ molecular mass standard are given across the top. The larger fraction numbers indicate the fraction with smaller molecular weight. (b) TET1 and SIN3A were shown interacting with hMOF using nuclear protein immunoprecipitations (IPs) in TET1 overexpressing HEK293T cells. (c) Schematic representation of TET1 fragments, including Flag-FL1, Flag-FL2, and Flag-FL3 (CXXC: binding CpG islands; CD: Cysteine-rich domain; DSBH: double stranded $\beta$-helix). (d) HEK293T cells were transiently transfected with Flag-FL1, Flag-FL2, and Flag-FL3, respectively. Nuclear protein IPs were performed using a Flag-tag antibody, followed by Western blot analysis using indicated antibodies. (e) GST and GST-FL3 were expressed in BL21 cells and purified following pGEX-GST-vector's manual. His-SIN3A and His-hMOF1 were also expressed in BL21 and purified and. Pull down assays were performed using a GST-tag antibody. (f) Co-transfections with Flag-TET1-FL and H4K16WT, H4K16Q, or H4K16R were carried out followed by IPs using a Flag-tag antibody. (g) Western blot analysis of the fractions in HA-H4K16R and HA-HK16WT overexpressing cells using antibodies as indicated. (h) Western blot analysis of the fractions before and after treatment with $1 \mu \mathrm{M}$ TSA for $1 \mathrm{~h}$ in HEK293T cells using antibodies as indicated.

\section{Figure 3. TET1 deficiency results in a reduction of H4K16ac levels}

(a) Western blot analysis of histone modifications in Tet ${ }^{-/}$MEF cells, TET1-knockdown HCT116 cells and TET1-knockdown HeLa cells using specific antibodies as indicated. (b) Immunofluorescence staining of Tet $1^{-/-}$MEF cells, TET1 knockdown HCT116 cells and TET1-knockdown HeLa cells with H4K16ac antibody. Nuclei were stained with DAPI. (c) Immunofluorescence staining of 53BP1 foci formation in Tet $1^{-/-}$MEF cells. Nuclei were stained with DAPI. (d) Statistical analysis of 53BP1 foci in Tet $1^{-1-}$ MEF cells and WT MEF cells. 
Figure 4. TET1 switches hMOF and/or SIRT1 binding on chromatin and regulates H4K16ac binding on the promoters of DNA repair genes

(a) Western blot analysis of the fractions in TET1-knockdown HEK293T cells using antibodies as indicated. (b) RT-qPCR analysis of TP53BP1, RAD51, RAD50, and BRCA1 mRNA levels in HA-hMOF overexpressing HEK293T cells, with or without TET1-knockdown, as indicated $\left({ }^{*} p<0.05,{ }^{* *} p<0.01,{ }^{* * *} p<0.001\right)$. (c) ChIP-qPCR analysis of $\mathrm{H} 4 \mathrm{~K} 16 \mathrm{ac}$ binding at the promoter of RAD50, BRCA1, RAD51, and TP53BP1 gene loci in HA-hMOF-overexpressing HEK293T cells, with or without TET1-knockdown, as indicated $\left({ }^{*} p<0.05,{ }^{* *} p<0.01,{ }^{* * *} p<0.001\right)$. (d) RT-qPCR analysis of RAD50, BRCA1, RAD51, and TP53BP1 mRNA levels in SIRT1-knockdown HEK293T cells, with or without TET1-knockdown, as indicated $\left({ }^{*} p<0.05,{ }^{* *} p<0.01,{ }^{* *} p<0.001\right)$. (e) ChIP-qPCR analysis of H4K16ac binding at the promoter of RAD50, BRCA1, RAD51, and TP53BP1 in SIRT1-knockdown HEK293T cells, with or without TET1-knockdown, as indicated $\left({ }^{*} p<0.05,{ }^{* *} p<0.01,{ }^{* * *} p<0.001\right)$. $p$ values were calculated by unpaired Student's $t$ test.

Figure 5. Depletion of TET1 impairs HRR and NHEJ and downregulation of DNA repair genes

(a) The phenotype of WT mice and Tet $1^{+-}$mice after 3Gy x-ray irradiation. Mice were irradiated with a single whole-body dose of $3 G y$ x-rays at 60 days of age. Concurrent sham-irradiated control groups were also examined from the same litter where possible to minimize genetic bias. (b) Statistical analysis of the coat-state condition of mice after 3Gy x-ray radiation, according to the method published by $\mathrm{C}$ Nasca, et al. (no x-ray: WT $n=14$, Tet $1^{+/-} n=15 ; x$-ray: WT, $n=13$, Tet $1^{+/-}, n=10 ;{ }^{*} p<0.05$, N.S $p>0.05$ ). (c) DNA damage in WT and Tet1 ${ }^{-/}$MEF cells, as measured by neutral comet assay. (d, e) Quantification of comet experiments (shown in a) the percentage of DNA in the comet tail or the tail moment was measured and statistical analysis was performed using GraphPad software. (f) DAPI staining and microscope analysis of micronuclei in Tet $1^{-/-}$MEF cells. (g) Statistical analysis of micronuclei in WT and Tet $1^{-/-}$MEF cells $\left({ }^{* *} p<0.01, \mathrm{~N}=\right.$ the number of the cells). (h) Frequency of HRR after TET1, TET2, or 
TET3 depletion $\left({ }^{* *} p<0.01\right)$. (i) Frequency of NHEJ after TET1, TET2, or TET3 depletion $\left({ }^{* *} p<0.01\right)$. (j) RT-qPCR analysis of mRNA levels in DNA repair genes (RAD50, BRCA1, RAD51, and TP53BP1) in TET1-knockdown cells ( ${ }^{*} p<0.05$, $\left.{ }^{* *} p<0.01\right)$. (k) Western blot analysis of 53BP1, RAD51, RAD50, and BRCA1 protein expression in TET1-knockdown HEK293T cells. $p$ value was calculated by unpaired Student's $t$ test.

Figure 6. Dynamic regulation of TET1, SIN3A, hMOF, and SIRT1 binding on chromatin in response to $\mathrm{H}_{2} \mathrm{O}_{2}$-induced oxidative damage

(a) Western blot analysis of histone modifications levels, using specific antibodies, after $1 \mu \mathrm{M}$ ADR, $1 \mu \mathrm{M}$ Bleo, $1 \mu \mathrm{M} \mathrm{CPT}$, and $2 \mathrm{mM} \mathrm{H}_{2} \mathrm{O}_{2}$ treatments for $1 \mathrm{~h}$, respectively. (b) Western blot analysis on chromatin fraction using antibodies as indicated after ADR, Bleo, CPT, and $\mathrm{H}_{2} \mathrm{O}_{2}$ treatments for $1 \mathrm{~h}$, respectively. $\gamma \mathrm{H} 2 \mathrm{AX}$ and $\mathrm{H} 2 \mathrm{AX}$ were used as a DNA damage marker and a chromatin fraction marker, respectively. GAPDH was used as a cytoplasm fraction marker. (c) HEK293T cells were transfected with Flag-FL3 and then untreated or treated with $\mathrm{H}_{2} \mathrm{O}_{2}$. IPs were performed using a Flag-tag antibody, followed by Western blot analysis using the indicated antibodies. (d) RT-qPCR analysis of mRNA levels in RAD50, BRCA1, RAD51, and TP53BP1 in untreated and $\mathrm{H}_{2} \mathrm{O}_{2}$-treated HEK293T cells $\left({ }^{*} p<0.05\right.$, $\left.{ }^{* *} p<0.01\right)$. (e) Smooth scatterplot of DNA methylation levels at the promoter of DNA repair genes in HEK293T cells, with or without $\mathrm{H}_{2} \mathrm{O}_{2}$ treatment, through RRBS. Pearson Correlation Coefficient $=1$. (f) IGV map of the DNA methylation levels at the TSS of RAD50, BRCA1, RAD51, and TP53BP1. Both the methylation level at promoters and flanking regions were displayed on two IGV tracks, one for the control group and the other for $\mathrm{H}_{2} \mathrm{O}_{2}$ treatment group in HEK293T cells. (g) ChIP-qPCR verification of TET1 and $\mathrm{H} 4 \mathrm{~K} 16 \mathrm{ac}$ binding before and after $\mathrm{H}_{2} \mathrm{O}_{2}$ treatment in HEK293T cells. $p$ value was calculated by unpaired Student's $t$ test.

Figure 7. Similar recomposition mechanism of the TET1/SIN3A/hMOF complex was observed in an oncogenic transformed cell line 
(a) RT-qPCR analysis of mRNA levels of 53BP1, RAD51, RAD50, and BRCA1 in T29 and $\mathrm{T} 29 \mathrm{H}$ cells, respectively $\left({ }^{*} p<0.05,{ }^{* *} p<0.01\right)$. (b) Western blot analysis of TET1, SIRT1, hMOF, and SIN3A bindings on chromatin in T29 and T29H cells, respectively. (c) Western blot analysis of histone modifications in $\mathrm{T} 29$ and $\mathrm{T} 29 \mathrm{H}$ cells using specific antibodies as indicated. (d, e) ChIP-qPCR analysis of both TET1 and H4K16ac binding on the promoter of RAD50, RAD51, BRCA1, and TP53BP1 in T29 and T29H cells $\left({ }^{*} p<0.05,{ }^{* *} p<0.01\right)$. (f) Working model of the dynamic regulation of TET1, SIN3A, hMOF, and SIRT1 via targeting H4K16ac in response to oxidative damage. 


\section{SUPPLEMENTARY MATERIAL}

Supplementary Figure 1. Enrichment analysis of Tet1, Sin3a, Mof, and H4K16ac Heat-map showed a ChIP-seq signal enrichment visualization of Tet1, Sin3a, Mof, and $\mathrm{H} 4 \mathrm{~K} 16 \mathrm{ac}$ in mESCs.

Supplementary Figure 2. Binding states of Tet1/Sin3a/Mof, PRC2/Suz12/Ezh2, and Sin3a/Hdac1/Hdac2 complexes and its function enrichment analysis in the promoter regions

(a) The seven binding states of TET1 as ordered by emission and transition parameters through ChromHMM software. (b) A Chow-Ruskey diagram of the overlapped genes of 5 binding states (M2, M3, M5, M6, and M7). (c) Top 60 biological processes and KEGG pathways of the 5 binding states. $p$ values were converted to $-10^{\star} \log (p$ value)

Supplementary Figure 3. ChIP-seq signals of Tet1, Sin3a, Mof, and H4K16ac in promoter regions of the DNA repair genes

IGV map presented ChIP-seq signals of Tet1, Sin3a, Mof, and H4K16ac in promoter regions of some DNA repair genes, including Brca1, Brca2, Rad50, Rad51, and MIh1.

\section{Supplementary Figure 4. Increased $\gamma \mathrm{H} 2 \mathrm{AX}$ in TET1-depleted cells}

(a) DNA damage marker $\gamma \mathrm{H} 2 \mathrm{AX}$ was stained in WT and Tet $1^{-1-}$ MEF cells. Nuclei were stained with DAPI. (b) Statistical analysis of $\gamma \mathrm{H} 2 \mathrm{AX}$ foci pictured in (a). More than ten number of foci was calculated as ten $\left({ }^{* *} p<0.01, N=\right.$ the number of the cells). (c) Western blot analysis of $\gamma \mathrm{H} 2 \mathrm{AX}$ level in TET1-, TET2-, or TET3-knockdown HEK293T cells.

Supplementary Figure 5. Binding of TET1 on chromatin is dependent on ATM

(a) Western blot analysis of the fractions before and after $1 \mu \mathrm{M}$ wortmannin treatment in HEK293T cells. (b) Western blot analysis of the fractions before and after $1 \mu \mathrm{M}$ CGK733 in HEK293T cells. (c) Western blot analysis of the fractions of the control 
and ATM siRNA-knockdown using indicated antibodies in HEK293T cells. (d) Western blot analysis of the fractions before and after $1 \mu \mathrm{M}$ DNA-PKcs inhibitors NU7026 treatment using indicated antibodies in HEK293T cells.

\section{Supplementary Figure 6. Analysis of DNA methylation on the promoter of the} DNA repair genes and oxidative damage levels in T29 and T29H cells

(a) DNA methylation levels of RAD50, MLH1, BRCA1, RAD51, and TP53BP1 genes in T29 and T29H cells through RRBS. (b) Dot blot analysis of oxidative damage level in $\mathrm{T} 29$ and $\mathrm{T} 29 \mathrm{H}$ cells using an 8-OH-dG antibody.

Supplementary Figure 7. Interactions between SIRT1 and PRC2 complex and binding of H3K9ac on the promoter of the DNA repair genes before and after $\mathrm{H}_{2} \mathrm{O}_{2}$ treatment

(a) Nuclear protein IPs were performed using SIRT1 antibody with or without $2 \mathrm{mM}$ $\mathrm{H}_{2} \mathrm{O}_{2}$ treatment and then detected by Western blot analysis using SUZ12 and EZH2 antibodies. (b) ChIP-qPCR analysis of H3K9ac binding on the promoter of RAD50, MLH1, RAD51, BRCA1, and TP53BP1 with or without $\mathrm{H}_{2} \mathrm{O}_{2}$ treatment in HEK293T cells (N.S $p>0.05)$.

Supplementary Table 1. Summary of published ChIP-seq data sets of DNA-binding proteins and histone modifications in mESCs

Supplementary Table 2. Occupancies of Tet1, Mof, Sin3a, Hdac1, Hdac2, H4K16ac, and H3K27me3 on the promoter of DNA repair genes from ChIP-seq data sets in mESCs 
Fig. 1

A

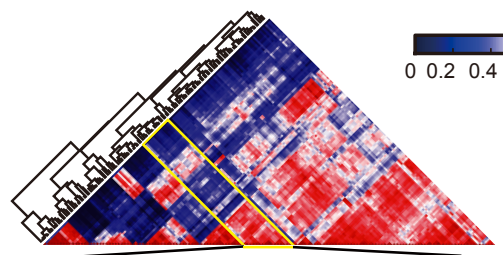

Tet1, Kdm2a, Dpy30, Mof, Sin3a, Lsd1, Oct4, P300, Dax1, Dmap1, Max, Nanog, Myc, Tip60

D

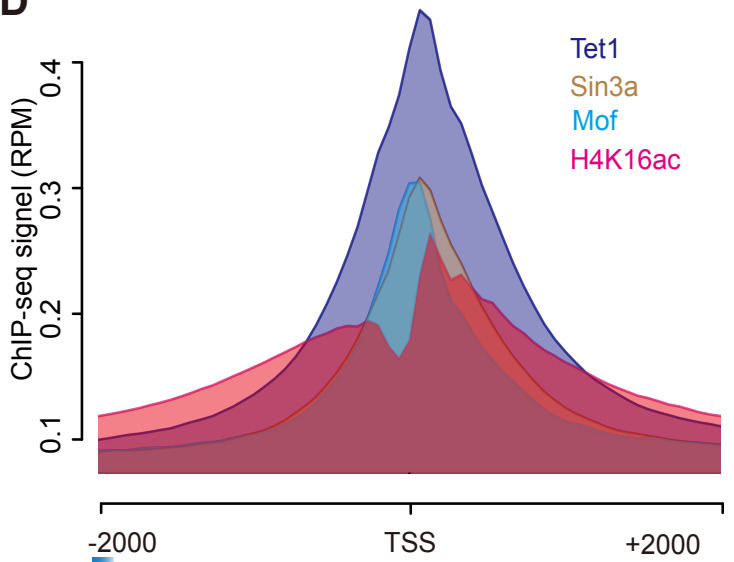

$\mathbf{F}$

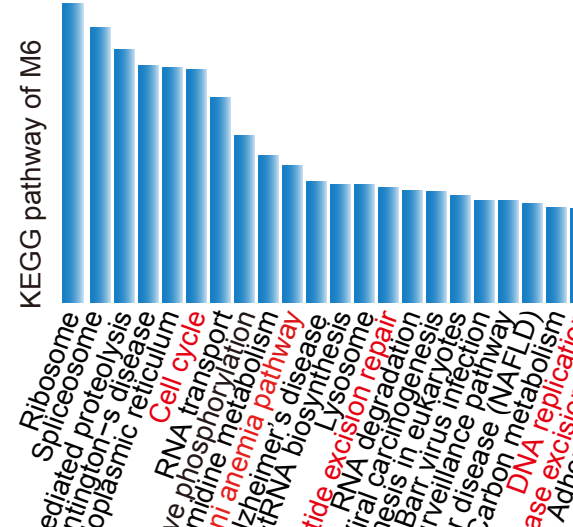

B

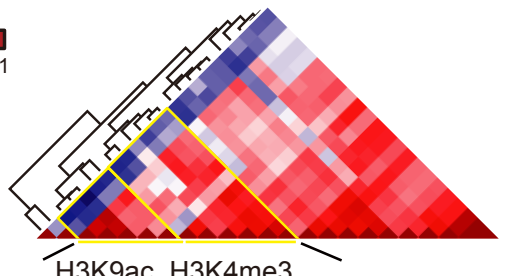

H4K16ac, H3K27ac, Tet1, Kdm2a,

Mof, Dpy30, Sin3a, Lsd1
C
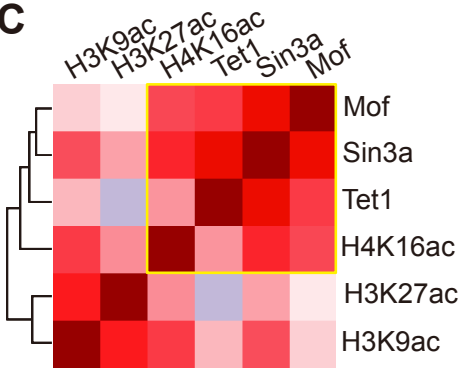
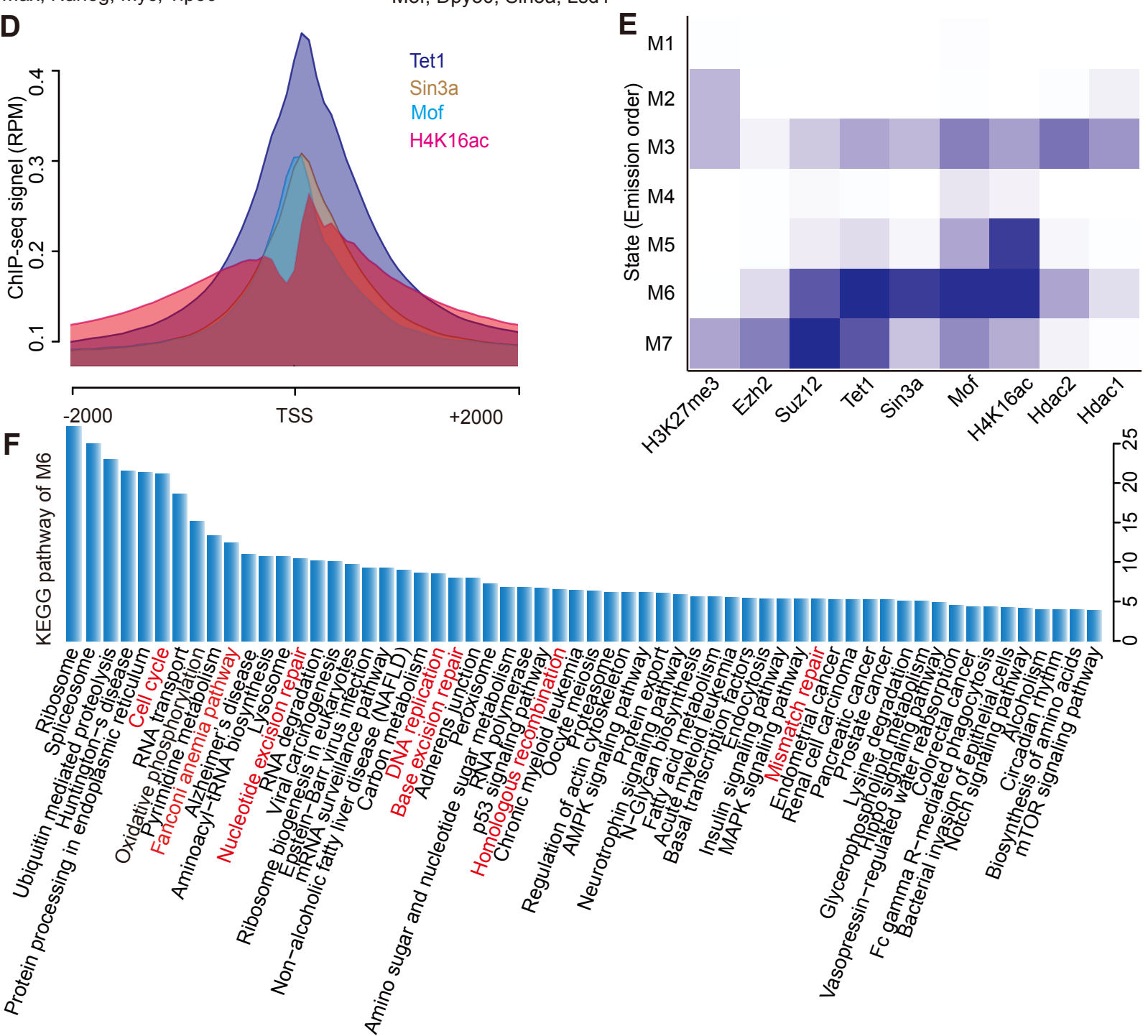

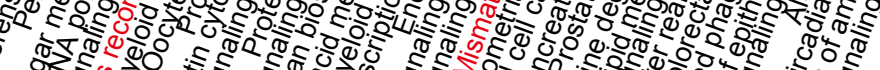

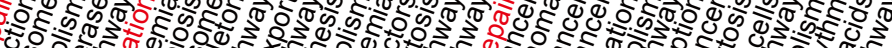

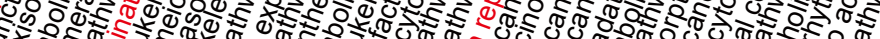

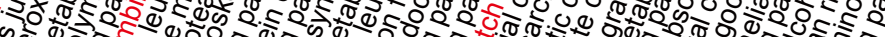

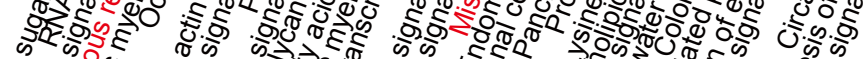

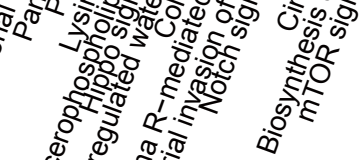
की के के 
Fig. 2

A

B

small complex

$12 \% \longrightarrow 30 \%$

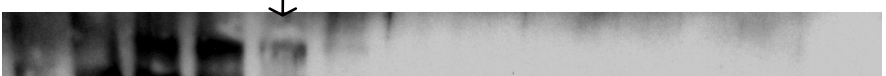

IB: TET1

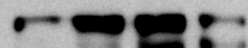

IB: SIN3A

IB: hMOF
C

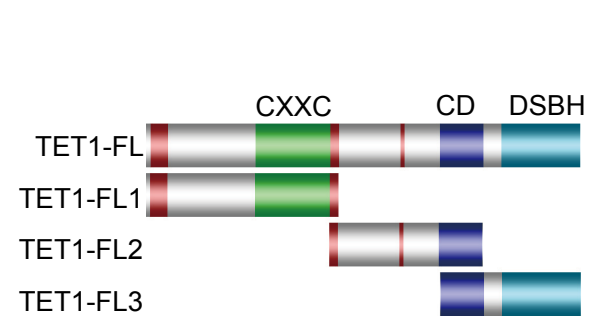

F

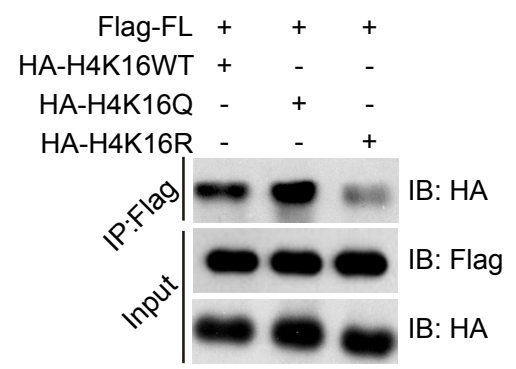

D

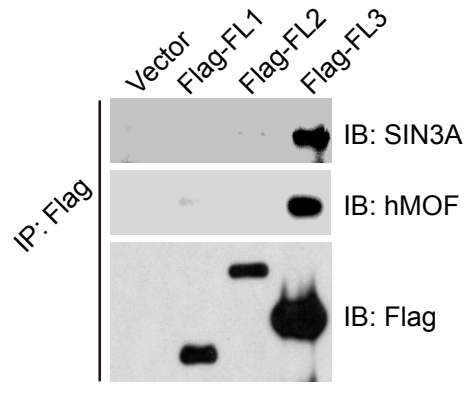

G

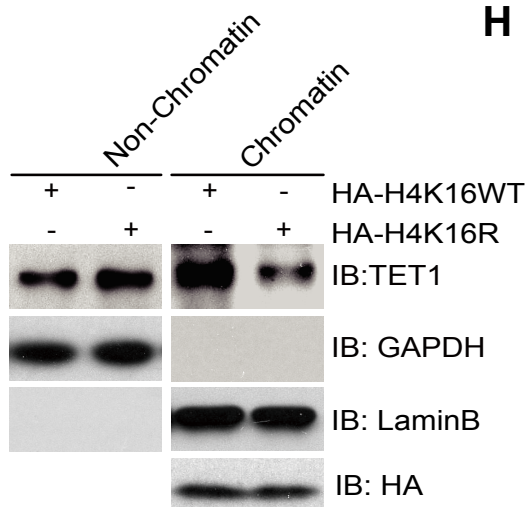

E
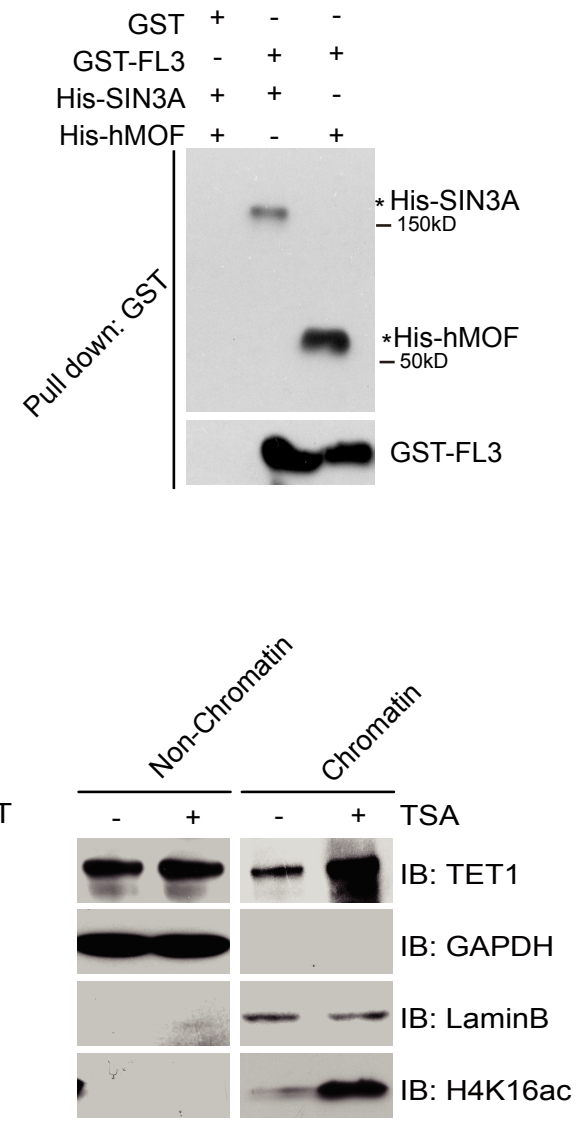
Fig. 3

A
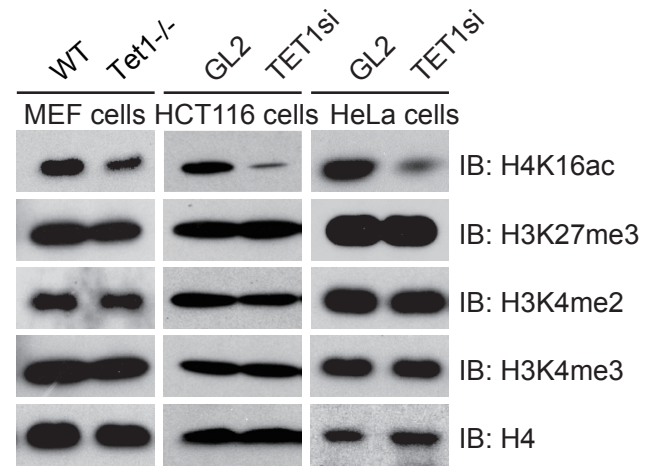

C

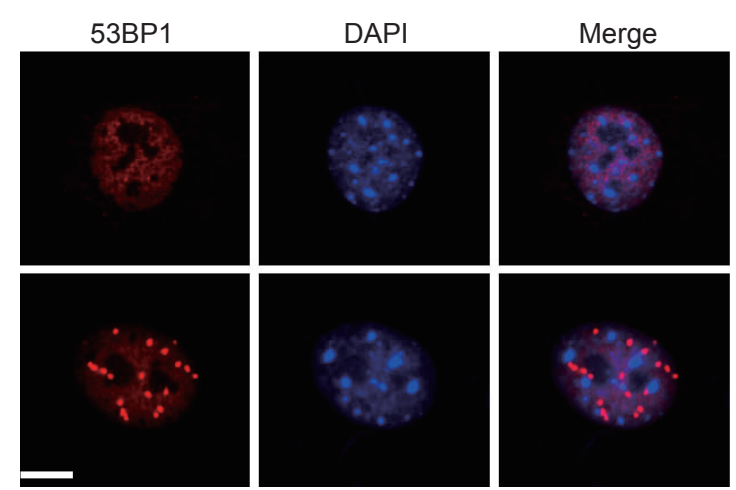

D

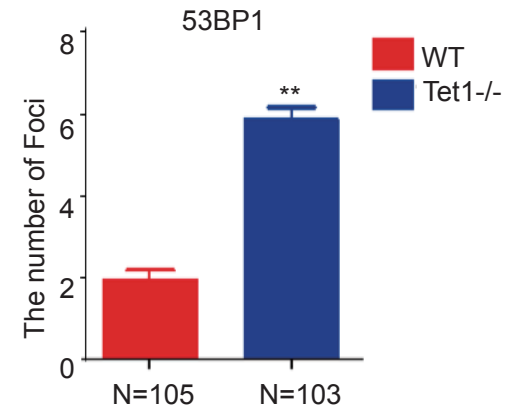

B
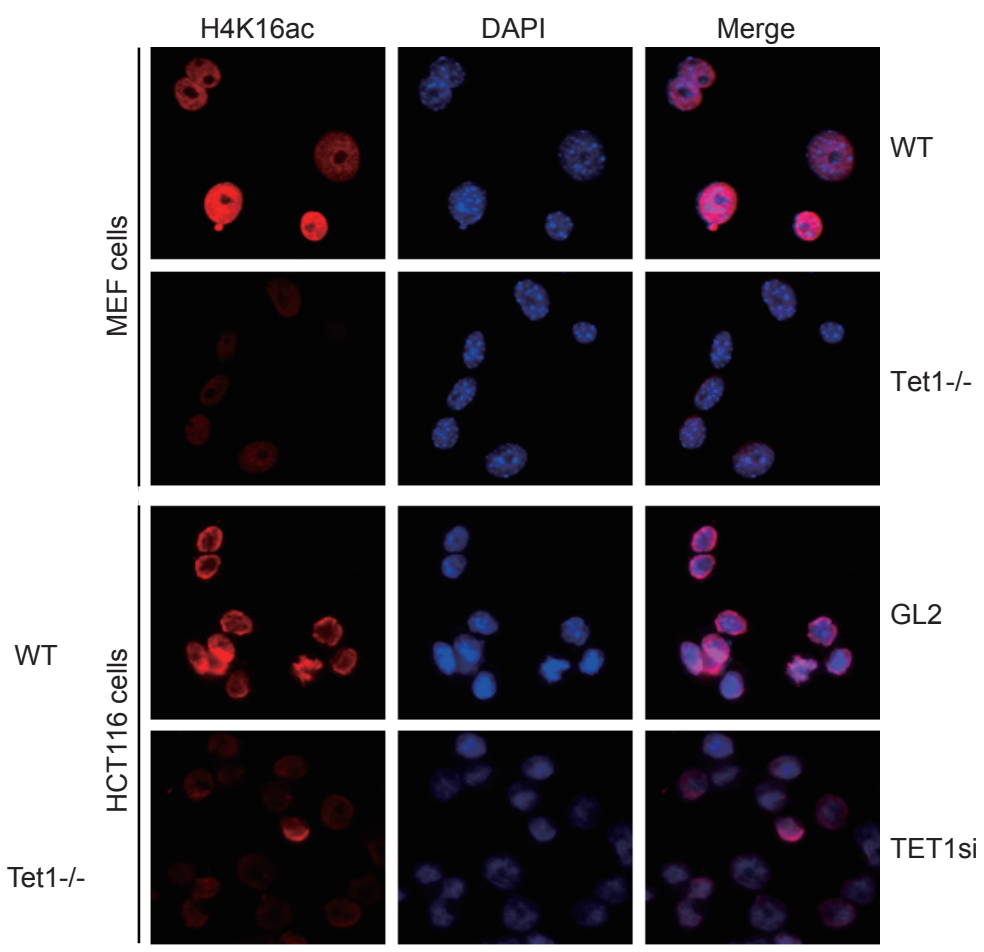

GL2
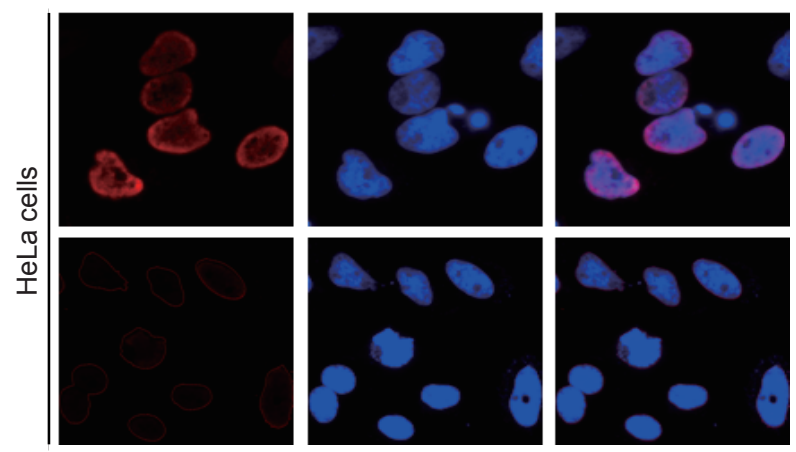

GL2

TET1si 
Fig. 4

A
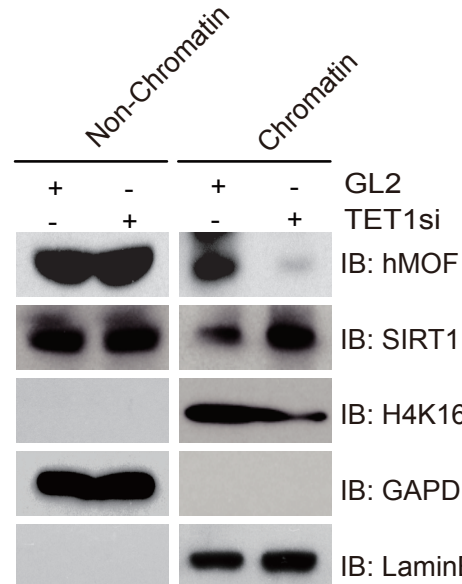

IB: SIRT1

IB: H4K16ac

IB: GAPDH

IB: LaminB
B
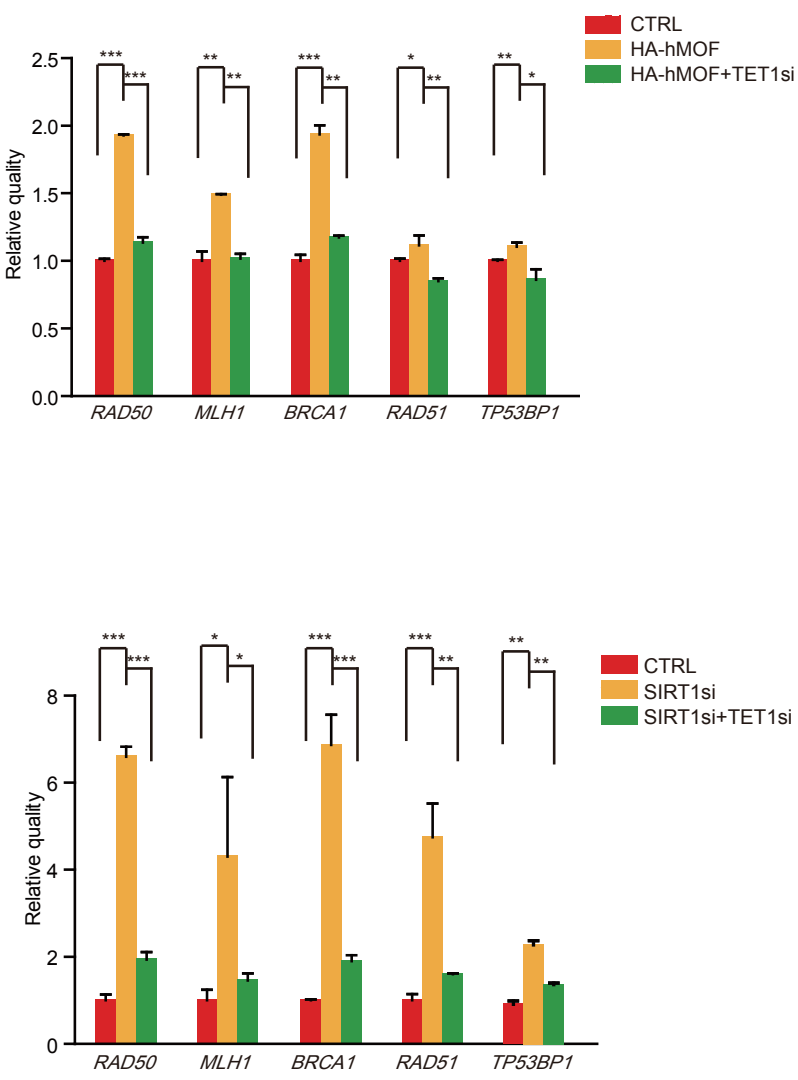

E

D

C

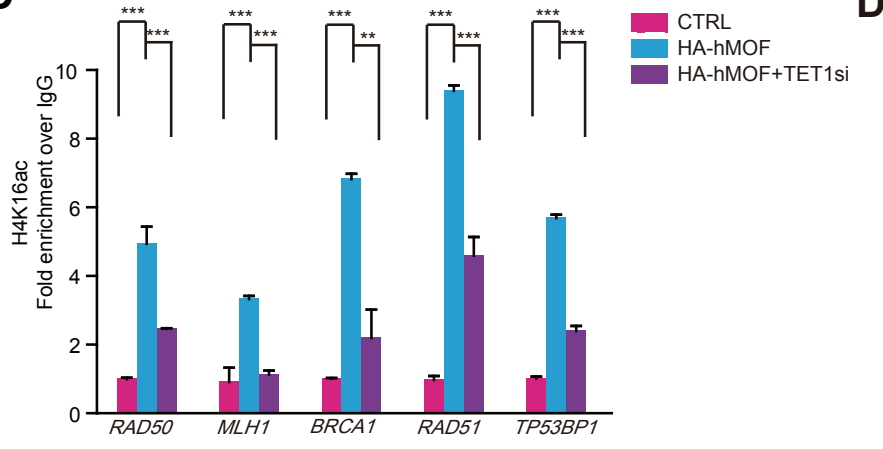

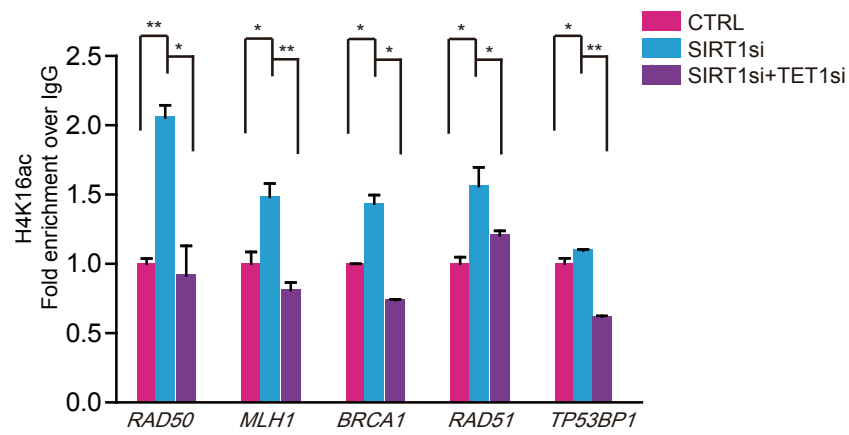


Fig. 5

A

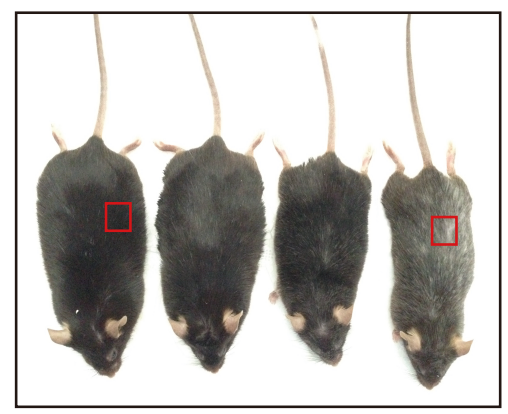

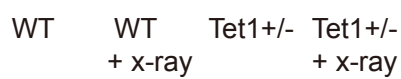

D
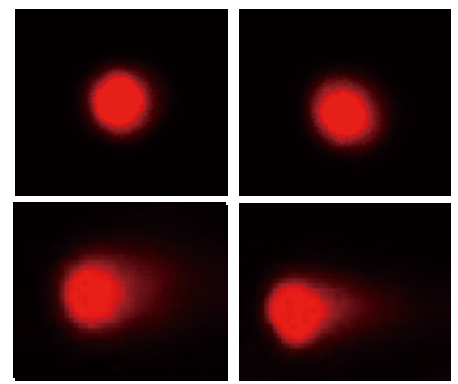

F

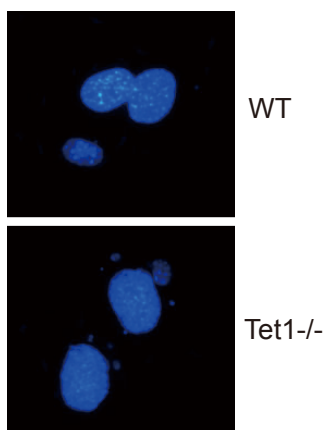

I

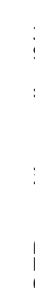

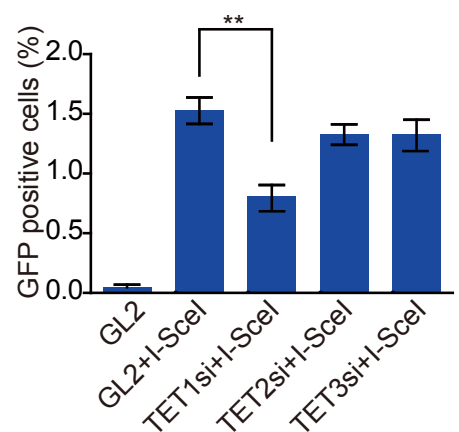

B

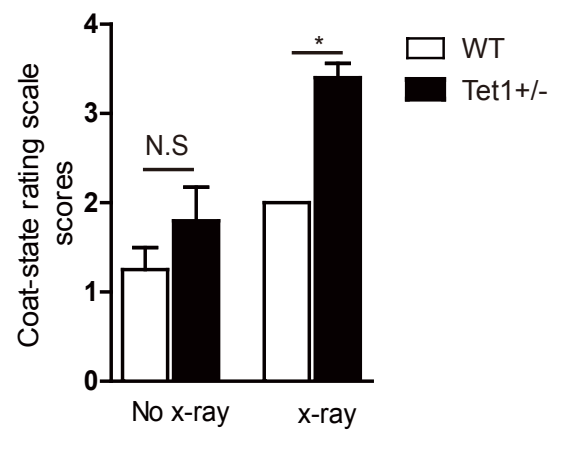

E

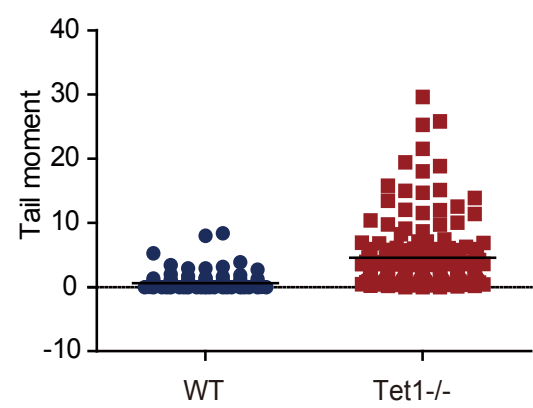

H
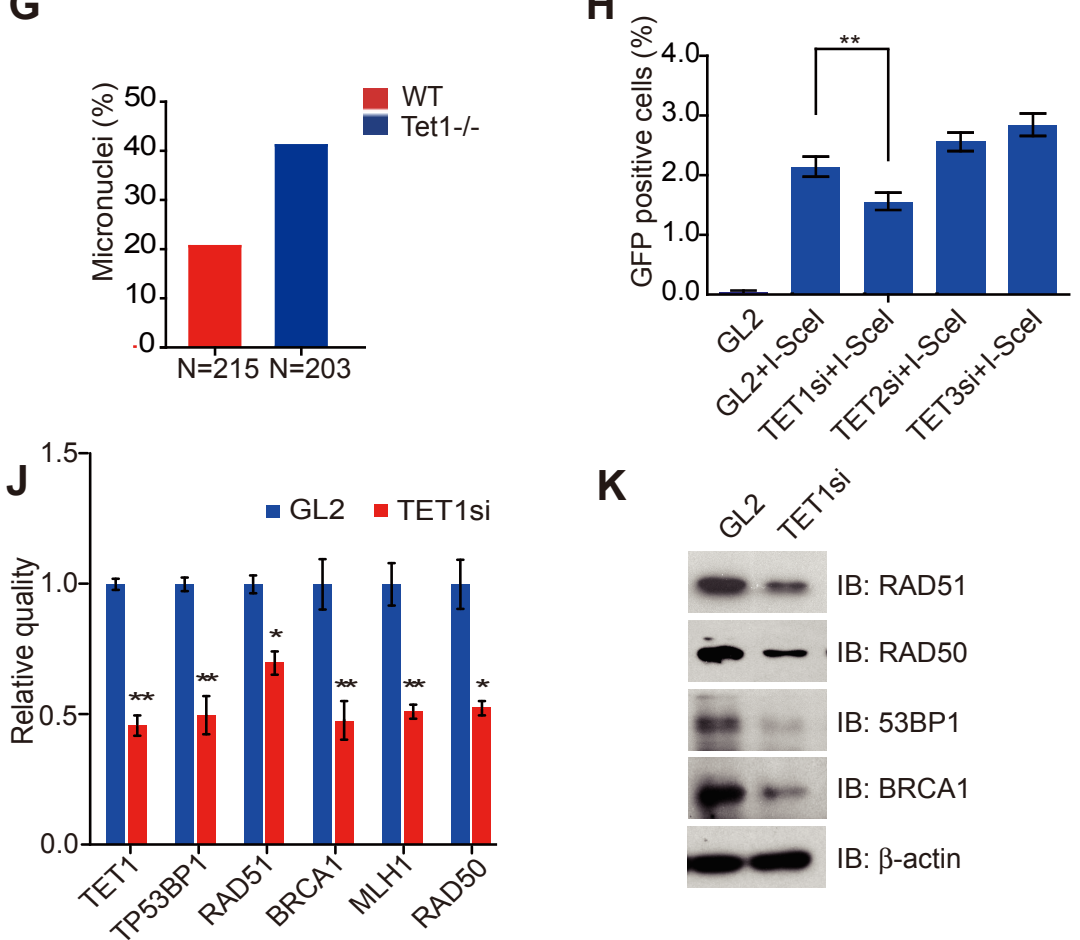

K

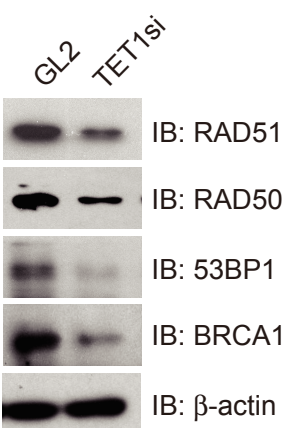


Fig. 6

A

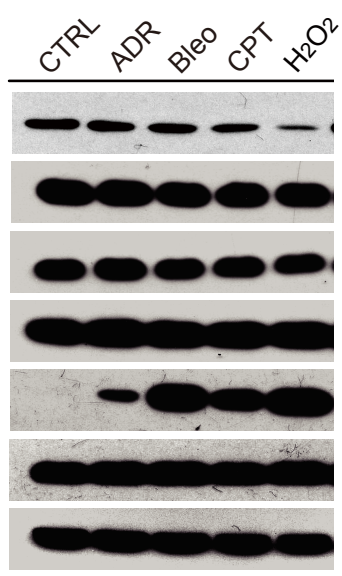

D

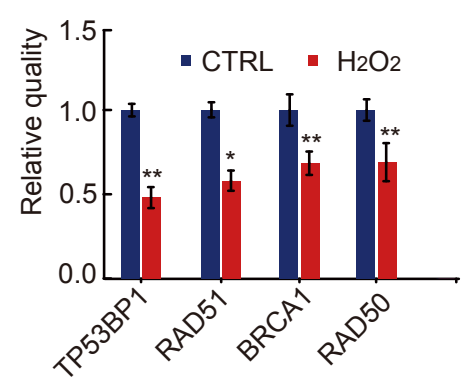

F
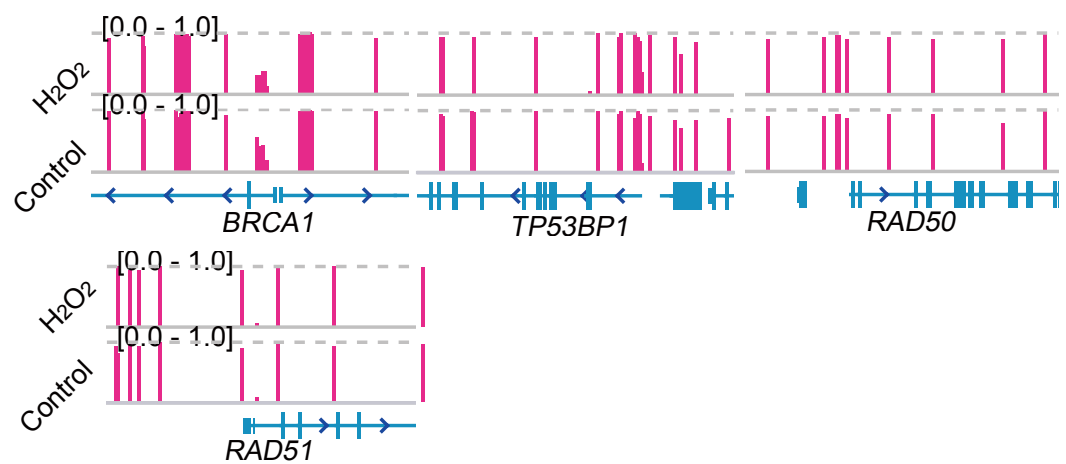

B

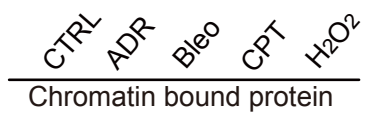

IB: H4K16ac

IB: H3K4me2

IB: H3K4me3

IB: H3K27me3

IB: H3

IB: $\beta$-actin
IB: $\gamma \mathrm{H} 2 \mathrm{AX}$

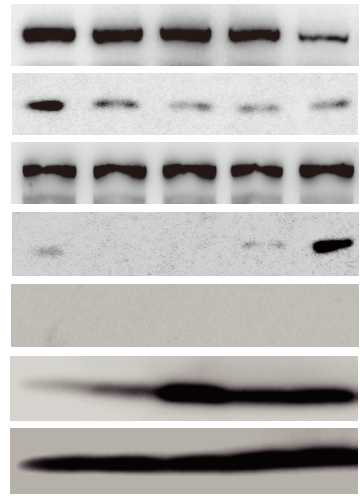

IB: TET1

IB: hMOF

IB: SIN3A

IB: SIRT1

IB: GAPDH

IB: $\gamma \mathrm{H} 2 \mathrm{AX}$

IB: $H 2 A X$
E

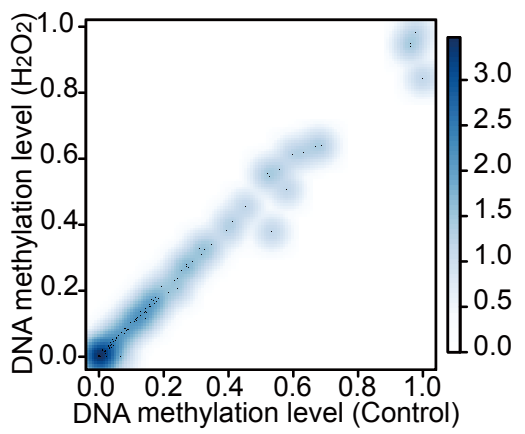

C

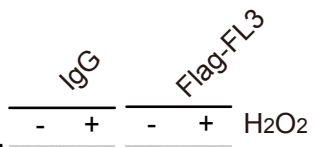

$\infty \quad \infty$ IB: SIN3A

$-\infty$ IB: hMOF

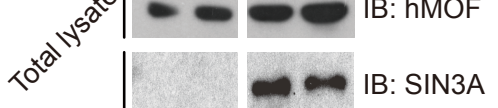

IB: hMOF

IB: Flag

G

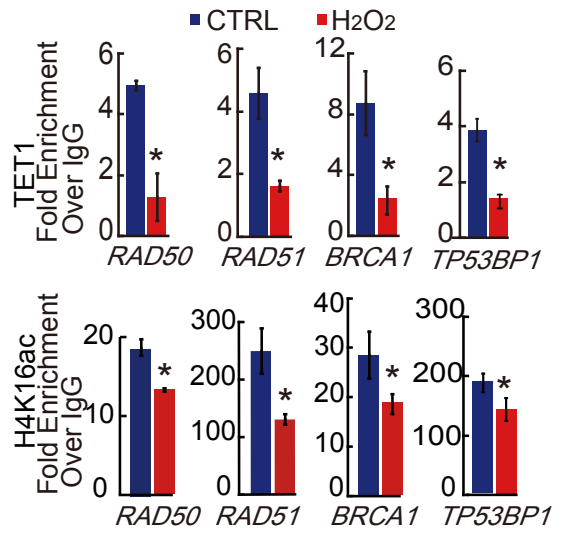


